\title{
openheart Myocardial fibrosis, diastolic dysfunction and elevated liver stiffness in the Fontan circulation
}

\author{
Tarek Alsaied (D) , ${ }^{1}$ Ryan A Moore, ${ }^{1}$ Sean M Lang, ${ }^{1}$ Vien Truong, ${ }^{2}$ \\ Adam M Lubert (iD , ${ }^{1}$ Gruschen R Veldtman (1) , ${ }^{1}$ Konstantin Averin, ${ }^{3}$ \\ Jonathan R Dillman, ${ }^{4}$ Andrew T Trout, ${ }^{4}$ Wojciech Mazur, ${ }^{2}$ Michael D Taylor, ${ }^{1}$ \\ Quan He, ${ }^{1}$ David LS Morales, ${ }^{1}$ Andrew N Redington, ${ }^{1}$ Bryan H Goldstein (D) ${ }^{1,5}$
}

\begin{abstract}
- Additional material is published online only. To view, please visit the journal online (http://dx.doi.org/10.1136/ openhrt-2020-001434)
\end{abstract}

To cite: Alsaied T, Moore RA, Lang SM, et al. Myocardial fibrosis, diastolic dysfunction and elevated liver stiffness in the Fontan circulation. Open Heart 2020;7:e001434. doi:10.1136/ openhrt-2020-001434

TA and RAM contributed equally.

Received 29 August 2020 Revised 23 September 2020 Accepted 6 0ctober 2020
Check for updates

(c) Author(s) (or their employer(s)) 2020. Re-use permitted under CC BY-NC. No commercial re-use. See rights and permissions. Published by BMJ.

For numbered affiliations see end of article.

Correspondence to Dr Tarek Alsaied; tarek.alsaied@ cchmc.org

\section{ABSTRACT}

Introduction Single ventricle diastolic dysfunction and hepatic fibrosis are frequently observed in patients with a Fontan circulation. The relationship between adverse haemodynamics and end-organ fibrosis has not been investigated in adolescents and young adults with Fontan circulation.

Methods Prospective observational study of Fontan patients who had a cardiac catheterisation. Cardiac MRI with T1 mapping was obtained to measure extracellular volume (ECV), a marker of myocardial fibrosis. Hepatic magnetic resonance elastography was performed to assess liver shear stiffness. Serum biomarkers of fibrosis including matrix metalloproteinases (MMPs) and tissue inhibitor of metalloproteinases (TIMPS) were measured. Very high ECV was defined as $>30 \%$ and elevated serum biomarkers as $>75$ th percentile for each biomarker. Results 25 Fontan patients ( $52 \%$ female) with mean age of $16.3 \pm 6.8$ years were included. Mean ECV was $28 \% \pm 5 \%$. There was a significant correlation between ECV and systemic ventricular end-diastolic pressure $(r=0.42$, $p=0.03$ ) and between ECV and liver stiffness ( $r=0.45$, $\mathrm{p}=0.05$ ). Patients with elevated ECV demonstrated elevations in MMPs and TIMPs. Similarly, patients with elevated MMPs and TIMPs had greater liver stiffness compared with patients with normal levels of these biomarkers.

Conclusions In Fontan patients, cardiac magnetic resonance evidence of myocardial fibrosis is associated with diastolic dysfunction, increased liver stiffness and elevated circulating biomarkers of fibrosis. These findings suggest the presence of a profibrotic milieu, with end-organ implications, in some patients with Fontan circulation.

\section{INTRODUCTION}

Adolescents and young adults with Fontan circulation may develop circulatory insufficiency and multiorgan dysfunction while remaining asymptomatic. ${ }^{12}$ Diastolic dysfunction is increasingly recognised as a clinical or subclinical driver of Fontan circulatory insufficiency or even Fontan failure. ${ }^{3}$ In the absence of validated non-invasive measures, direct

\section{Key questions}

What is already known about this subject?

- Diastolic dysfunction and Fontan-associated liver disease due to liver fibrosis are important morbidities in patients with a Fontan circulation.

What does this study add?

- This paper found an association between extracellular volume, a measure of myocardial fibrosis by cardiac MRI (CMR), Fontan and ventricular end-diastolic pressure and liver stiffness.

- This paper found an association of serum fibrosis biomarkers and both extracellular volume and liver stiffness.

- This may suggest the presence of a profibrotic milieu in some Fontan patients. This study gives mechanistic insights into Fontan liver disease and diastolic dysfunction.

How might this impact on clinical practice?

- This manuscript highlights the importance of CMR as an imaging tool in patients with single ventricle to detect interstitial fibrosis, which is associated with ventricular end-diastolic pressure. As CMR is now a routinely used tool in patients with single ventricle, the addition of tissue mapping will likely give more insights in this patient population. This can be easily implemented in clinical practice without adding significant burden to the clinical test.

invasive ventricular and Fontan circuit pressure measurement, with or without manoeuvres to unmask occult circulatory dysfunction, is the gold standard to diagnose diastolic dysfunction. ${ }^{4}$ Fibrosis is a common final pathway in chronic myocardial disease, including in diastolic heart failure. ${ }^{5}$ In adults with heart failure with preserved ejection fraction (HFpEF), myocardial fibrosis is intrinsically linked to ventricular stiffness and adverse remodelling. ${ }^{6}$ In Fontan patients, liver fibrosis is recognised as a nearly ubiquitous and time-dependent end-organ complication of the circulation and 
may be worsened in the setting of diastolic dysfunction and elevated Fontan pressure. ${ }^{2}$ Thus, myocardial and hepatic fibrosis may offer a biologically plausible explanation for the common phenotype observed in many adult Fontan patients: diastolic dysfunction and Fontan-associated liver disease.

Myocardial extracellular volume (ECV) fraction measured by cardiac MRI (CMR) is a validated noninvasive measure of interstitial myocardial fibrosis and is associated with diastolic dysfunction in other patient populations. ${ }^{78}$ Further, myocardial ECV has been shown to be elevated in Fontan patients. ${ }^{9}$ Similarly, liver shear stiffness assessed by liver magnetic resonance elastography (MRE) is a non-invasive surrogate for liver fibrosis and has been shown to be elevated in Fontan patients. ${ }^{10}$ To date, the association of myocardial ECV, liver stiffness and Fontan haemodynamics has not been evaluated. In this study, we sought to investigate the relationships between invasive haemodynamics, myocardial ECV, liver stiffness (a surrogate for hepatic fibrosis) and circulating biomarkers of fibrosis.

\section{METHODS}

\section{Patients}

Informed consent was obtained from study participants or their parents/guardians. Assent was obtained from patients older than 11 years. Adolescents and adults with Fontan circulation who underwent cardiac catheterisation with rapid volume expansion testing between
2015 and 2017 were consented to undergo a CMR and to measure fibrosis laboratory biomarkers. Potential participants with contraindication to CMR were excluded. Patients were involved in the design of the study through the Children's Heart Association of Cincinnati.

\section{Cardiac MRI}

CMR examinations were performed on 1.5-Tesla scanners (Ingenia; Philips Healthcare, Best, Netherlands). A gadolinium-based contrast agent, macrocyclic, intravenous contrast (gadoterate meglumine; Dotarem; Guerbet LLC, Princeton, New Jersey, USA) was administered at a dose of $0.2 \mathrm{mmol} / \mathrm{kg}$. Late gadolinium enhancement (LGE) images were acquired using a standard inversion recovery gradient echo pulse sequence.

T1 maps were acquired with a breath-hold modified Look-Locker inversion recovery sequence in the shortaxis plane at the midventricular level. ECV was measured from $\mathrm{T} 1$ maps before and $10 \mathrm{~min}$ postcontrast, as previously described. ${ }^{7}$ A blood sample was obtained at the time of CMR for measurement of haematocrit.

Cardiac ECV was calculated using the formula:

$$
\mathrm{ECV}=(1-\text { Haematocrit }) \mathrm{x} \frac{\frac{1}{\text { postcontrast myocardial T1 }}-\frac{1}{\text { native myocardial T1 }}}{\frac{1}{\text { postcontrast blood } \mathrm{T} 1}-\frac{1}{\text { native blood T1 }}}
$$

Analysis was performed using commercially available software (Cvi42, Circle Cardiovascular Imaging, Calgary, Canada). Myocardial contours were manually drawn by a paediatric cardiologist with 5-year experience in CMR. The ventricular free wall of the single ventricle was

Table 1 Demographic and clinical characteristics of the study population

\begin{tabular}{|c|c|c|c|c|}
\hline \multirow[b]{2}{*}{ All patients } & \multirow{2}{*}{$\begin{array}{l}\text { Total } \\
n=25\end{array}$} & \multicolumn{2}{|c|}{ Myocardial fibrosis } & \multirow[b]{2}{*}{$P$ value } \\
\hline & & DMF+ $(n=12)$ & DMF- $(n=13)$ & \\
\hline \multicolumn{5}{|l|}{ Gender } \\
\hline Age at Fontan (years) & $4.1 \pm 3.4$ & $44.0 \pm 2.7$ & $4.1 \pm 1.6$ & 0.9 \\
\hline Age at CMR (years) & $18.5 \pm 6.9$ & 0.67 & $19.0 \pm 6.9$ & 0.67 \\
\hline Time between Fontan and catheterisation (years) & $13.0 \pm 6.3$ & $13.2 \pm 6.5$ & $13.2 \pm 6.5$ & 0.78 \\
\hline Time between catheterisation and CMR (years) & $1.8 \pm 1.3$ & $1.7 \pm 1.6$ & $1.7 \pm 1.6$ & 0.57 \\
\hline Ventricular morphology & & & & 0.61 \\
\hline Left & $18(72 \%)$ & 9 & 9 & \\
\hline Right & $6(24 \%)$ & 3 & 3 & \\
\hline History of plastic bronchitis & 2 & 1 & 1 & NA \\
\hline Type of Fontan & & & & 0.80 \\
\hline Atriopulmonary & $2(8 \%)$ & 1 & 1 & \\
\hline Lateral tunnel & $13(52 \%)$ & 7 & 6 & \\
\hline Extracardiac conduit & $10(40 \%)$ & 4 & 6 & \\
\hline
\end{tabular}

Results are presented as mean \pm SD or frequency (\%).

CMR, cardiac MRI; DMF, diffuse myocardial fibrosis. 
Table 2 Observed findings from catheterisation, CMR and MRE in patients with and without diffuse myocardial fibrosis

\begin{tabular}{|c|c|c|c|c|}
\hline & Total & Myocardial fib & & \\
\hline & $(n=25)$ & $D M F+(n=12)$ & DMF- $(n=13)$ & $P$ value \\
\hline Ejection fraction (\%, CMR) & $54 \pm 7$ & $56 \pm 5$ & $53 \pm 8$ & 0.17 \\
\hline Haematocrit (\%) & $45 \pm 4$ & $45 \pm 5$ & $45 \pm 4$ & 0.89 \\
\hline Native $\mathrm{T} 1$ values & $990 \pm 84$ & $1025 \pm 60$ & $958 \pm 93$ & 0.04 \\
\hline Baseline Fontan pressure $(\mathrm{mm} \mathrm{Hg})$ & $12 \pm 2$ & $13 \pm 3$ & $11 \pm 1$ & 0.02 \\
\hline Baseline ventricular end-diastolic pressure $(\mathrm{mm} \mathrm{Hg})$ & $9 \pm 2$ & $10 \pm 2$ & $8 \pm 2$ & 0.03 \\
\hline Baseline pulmonary vascular resistance (iWu) & $1.2 \pm 0.5$ & $1.3 \pm 0.6$ & $1.2 \pm 0.4$ & 0.55 \\
\hline Baseline aortic saturation (\%) & $93 \pm 3$ & $92 \pm 3$ & $94 \pm 2$ & 0.13 \\
\hline Baseline cardiac index $\left(\mathrm{L} / \mathrm{min} / \mathrm{m}^{2}\right)$ & $3.4 \pm 0.8$ & $3.3 \pm 0.7$ & $3.4 \pm 0.9$ & 0.7 \\
\hline Change in ventricular EDP after stress $(\mathrm{mm} \mathrm{Hg})$ & $4.3 \pm 2.1$ & $4.4 \pm 0.6$ & $4.1 \pm 0.6$ & 0.69 \\
\hline Change in Fontan pressure after stress $(\mathrm{mm} \mathrm{Hg})$ & $3.4 \pm 1.9$ & $3.7 \pm 2.2$ & $3.0 \pm 1.3$ & 0.33 \\
\hline Change in cardiac output after stress $\left(\mathrm{L} / \mathrm{min} / \mathrm{m}^{2}\right)$ & $0.0 \pm 0.9$ & $-0.1 \pm 0.2$ & $0.1 \pm 0.3$ & 0.65 \\
\hline Stress Fontan pressure (mm Hg) & $15 \pm 3$ & $17 \pm 3$ & $14 \pm 1$ & 0.008 \\
\hline Stress ventricular end-diastolic pressure ( $\mathrm{mm} \mathrm{Hg}$ ) & $13 \pm 3$ & $14 \pm 3$ & $12 \pm 2$ & 0.04 \\
\hline Stress pulmonary vascular resistance (iWu) & $1.4 \pm 0.6$ & $1.5 \pm 0.7$ & $1.2 \pm 0.5$ & 0.28 \\
\hline Stress cardiac index $\left(\mathrm{L} / \mathrm{min} / \mathrm{m}^{2}\right)$ & $3.3 \pm 1.2$ & $3.1 \pm 1.0$ & $3.5 \pm 1.3$ & 0.44 \\
\hline Liver Stiffness $(\mathrm{n}=17)$ & $4.1 \pm 0.7$ & $4.5 \pm 0.3(n=10)$ & $3.4 \pm 0.7(n=7)$ & 0.066 \\
\hline
\end{tabular}

Results are presented as mean \pm SD or frequency (\%). $P$ values in bold are statistically significant.

CMR, cardiac MRI; EDP, end-diastolic pressure; iWu, indexed Wood units; MRE, magnetic resonance elastography.

contoured; the intraventricular septum was not assessed due to the difficulty in differentiating the septum of the hypoplastic ventricle as previously described. ${ }^{9}$ To ensure accurate $\mathrm{T} 1$ measurements and exclusion of the blood pool signal, regions of interest (ROIs) were drawn conservatively, keeping approximately $75 \%$ of the myocardial thickness within the ROI and excluding the blood pool, papillary muscles, chordae and trabeculations.

As ECV may vary depending on the sequence used and the CMR vendor, it is recommended to have institutional normal values. Our institutional normal values of ECV in patients without heart disease are $26 \% \pm 2 \%($ mean $\pm \mathrm{SD}){ }^{7}$ Patients with ECV $>28 \%$ (more than one SD above the mean) were grouped as diffuse myocardial fibrosis $(\mathrm{DMF}+)$, whereas those with ECV $\leq 28 \%$ were grouped as not having diffuse myocardial fibrosis (DMF-). A second cut point of ECV $>30 \%$ (two SD above the mean) was used to define patients with a very high ECV.

\section{Cardiac catheterisation}

Cardiac catheterisation was performed according to a standard institutional clinical Fontan protocol, with acquisition of comprehensive right and left heart haemodynamics. Oxygen consumption was directly measured in the catheterisation laboratory, when possible, and otherwise assumed. ${ }^{11}$ Following assessment of complete baseline haemodynamics, and prior to administration of radiographic contrast material, rapid volume expansion was performed by delivering $15 \mathrm{~mL} / \mathrm{kg}$ of normal saline briskly via central venous access (typically administered over $2-5 \mathrm{~min}$ ). Following a $5 \mathrm{~min}$ equilibration period, 'stress' haemodynamics were obtained. ${ }^{4}$ Invasive haemodynamic data collected included: Fontan pathway pressure that is measured in the Fontan conduit or Fontan baffle, ventricular end-diastolic pressures (EDPs), aortic saturation and pulmonary vascular resistance.

\section{Biomarker analysis}

Blood for serum biomarker analysis was obtained at the time of CMR. Plasma growth differentiation factor 15 (GDF-15) and galectin-3 were determined with Quantikine kits from R\&D systems (Minneapolis, Minnesota, USA); cross-linked carboxy-terminal telopeptide of type I collagen (CITP) and procollagen type I C-terminal propeptide (PICP) were assayed with Elisa kits from $\mathrm{MyBi}$ oSource (San Diego, California, USA) and matrix metalloproteinases (MMPs) and tissue inhibitor of metalloproteinases (TIMPs) were quantified with multiplex Quantibody Human MMP Array 1 from RayBiotech (Norcross, Georgia, USA).$^{12}$ As there are no previously published normal values for these biomarkers in the Fontan population, subjects were categorised as having high levels of a biomarker when in the top quartile ( $>75$ th percentile) for each biomarker.

\section{Liver MRE}

Liver MRE was obtained for clinical purposes in some of the study population. For all MRE examinations, patients were kept NPO (nothing by mouth) for at least 6 hours before the examination. MRE was performed on 1.5 T MRI Philips scanners. The details of our institutional protocol have been previously described. ${ }^{13}$ 
Table 3 Association of ECV with clinical, catheterisation and $M R I$ variables

\begin{tabular}{|c|c|c|}
\hline All patients $(n=25)$ & $\begin{array}{l}\text { Pearson } \\
\text { correlation } \\
\text { coefficient } \\
\text { with ECV }\end{array}$ & $P$ value \\
\hline Weight & 0.01 & 0.73 \\
\hline Age at CMR (years) & 0.02 & 0.47 \\
\hline Age at catheterisation (years) & 0.02 & 0.42 \\
\hline Time between Fontan and CMR (years) & 0.02 & 0.46 \\
\hline $\begin{array}{l}\text { Time between catheterisation and CMR } \\
\text { (years) }\end{array}$ & 0.02 & 0.42 \\
\hline Heart rate at CMR & 0.08 & 0.23 \\
\hline Liver stiffness & 0.45 & 0.05 \\
\hline End-diastolic volume & 0.04 & 0.39 \\
\hline End-systolic volume & 0.07 & 0.29 \\
\hline Ventricular ejection fraction & 0.02 & 0.53 \\
\hline Fontan type & & $0.66^{*}$ \\
\hline Ventricular morphology & & $0.46^{*}$ \\
\hline Baseline Fontan pressure (mm Hg) & 0.42 & 0.03 \\
\hline $\begin{array}{l}\text { Baseline ventricular end-diastolic pressure } \\
(\mathrm{mm} \mathrm{Hg})\end{array}$ & 0.44 & 0.03 \\
\hline Baseline pulmonary vascular resistance (iWu) & 0.01 & 0.68 \\
\hline Baseline cardiac index $\left(\mathrm{L} / \mathrm{min} / \mathrm{m}^{2}\right)$ & 0.06 & 0.37 \\
\hline Stress Fontan pressure (mm Hg) & 0.45 & 0.02 \\
\hline $\begin{array}{l}\text { Stress ventricular end-diastolic pressure } \\
(\mathrm{mm} \mathrm{Hg})\end{array}$ & 0.53 & 0.007 \\
\hline Stress pulmonary vascular resistance (iWu) & 0.07 & 0.26 \\
\hline Stress cardiac index $\left(\mathrm{L} / \mathrm{min} / \mathrm{m}^{2}\right)$ & 0.07 & 0.23 \\
\hline
\end{tabular}

*Wilcoxon test $p$ value. Bold numbers indicate $p$ value $\leq 0.05$. CMR, cardiac MRI; ECV, extracellular volume; iWu, indexed Wood units.

\section{Statistical analysis}

Two-sided Student's t-test or Wilcoxon rank-sum test were used to compare two groups of continuous variables if they were normally or non-normally distributed, respectively. Fisher's exact test was used to compare categorical variables. Univariate associations between normally distributed variables were estimated using the Pearson correlation coefficient $(\mathrm{r})$, while Spearman rank-order correlation (rho) was used for non-normally distributed data. To study the association between fibrosis laboratory biomarkers and ECV, Fisher's exact test was used to assess the prevalence of elevated biomarkers in the group with DMF+ orvery high ECV. To study the association of the laboratory biomarkers with liver stiffness, liver stiffness was compared between patients with and without the elevated biomarker. A twotailed $p$ value of $\leq 0.05$ was considered statistically significant. Statistical analyses were performed using JMP.

\section{RESULTS}

A total of 25 Fontan patients were included in the study. Mean age at the time of catheterisation with haemodynamic evaluation was $16.3 \pm 6.8$ years, and 13 patients $(52 \%)$ were female (table 1$)$. The mean duration of Fontan circulation, at the time of cardiac catheterisation, was $13.0 \pm 6.3$ years. Lateral tunnel was the most common Fontan type (52\%), and the majority of patients $(72 \%)$ had a single left ventricle. No patient had a fenestration at the time of the CMR.

\section{Observed CMR and haemodynamic data}

CMR and catheterisation-derived haemodynamic data are detailed in table 2 . The mean ECV was $28 \% \pm 5 \%$. No patient had LGE. The mean ventricular ejection fraction was $54 \% \pm 7 \%$. At catheterisation, the baseline Fontan pressure was $12 \pm 2 \mathrm{~mm} \mathrm{Hg}$, and the baseline ventricular EDP was $9 \pm 2 \mathrm{~mm} \mathrm{Hg}$. The baseline cardiac index was $3.4 \pm 0.8 \mathrm{~L} / \mathrm{min} /$ $\mathrm{m}^{2}$, and the pulmonary vascular resistance was $1.2 \pm 0.5$ index Wood units. Following rapid volume expansion testing, mean stress Fontan pressure was $15 \pm 3 \mathrm{~mm} \mathrm{Hg}$, and mean stress ventricular EDP was $13 \pm 3 \mathrm{~mm} \mathrm{Hg}$.

\section{Associations with cardiac ECV and clinical factors}

Cardiac ECV had no significant correlation with heart rate at CMR, ventricular end-diastolic volume or end-systolic volume, or ventricular ejection fraction (table 2). There was no significant difference between patients with single right ventricle and single left ventricle (28.6 \pm 3.0 vs $27.5 \pm 3.3$, $\mathrm{p}=0.46)$ in ECV. Twelve patients $(48 \%)$ were DMF+. There was no significant difference in any of the baseline characteristics between the patients categorised as DMF+ and DMF-.

\section{Associations with cardiac ECV and haemodynamic measures}

As demonstrated in table 3, there was a significant correlation between cardiac ECV and Fontan pressure $(\mathrm{r}=0.44$, $\mathrm{p}=0.03$ ) as well as EDP $(\mathrm{r}=0.42, \mathrm{p}=0.03$ ) (figures 1 and 2). A stronger correlation was appreciated between ECV and stress Fontan pressure $(\mathrm{r}=0.53, \mathrm{p}=0.007)$ and stress EDP $(r=0.45, p=0.02)$. There was no correlation between ECV and cardiac index or pulmonary vascular resistance at baseline or after rapid volume expansion (table 3 and figure 1). Following stratification by ECV\%, patients categorised as DMF+ had significantly higher Fontan and ventricular EDPs, both at baseline and with stress, compared with the DMF- patients (table 2 and figure 2).

\section{Myocardial fibrosis and serum fibrosis biomarkers}

Patients categorised as DMF+ did not demonstrate significant differences in circulating levels of serum fibrosis biomarkers when compared with DMF- patients (online supplemental table 1). However, a significantly higher proportion of patients with very high ECV demonstrated elevated MMP1, MMP2, MMP3, MMP 8, MMP10, MMP13, TIMP 1 and TIMP4 levels compared with the remainder of the cohort (figure 3 , online supplemental table 2). No association was found between ECV and serum biomarkers when each was evaluated as a continuous measure (online supplemental table 1 ). 

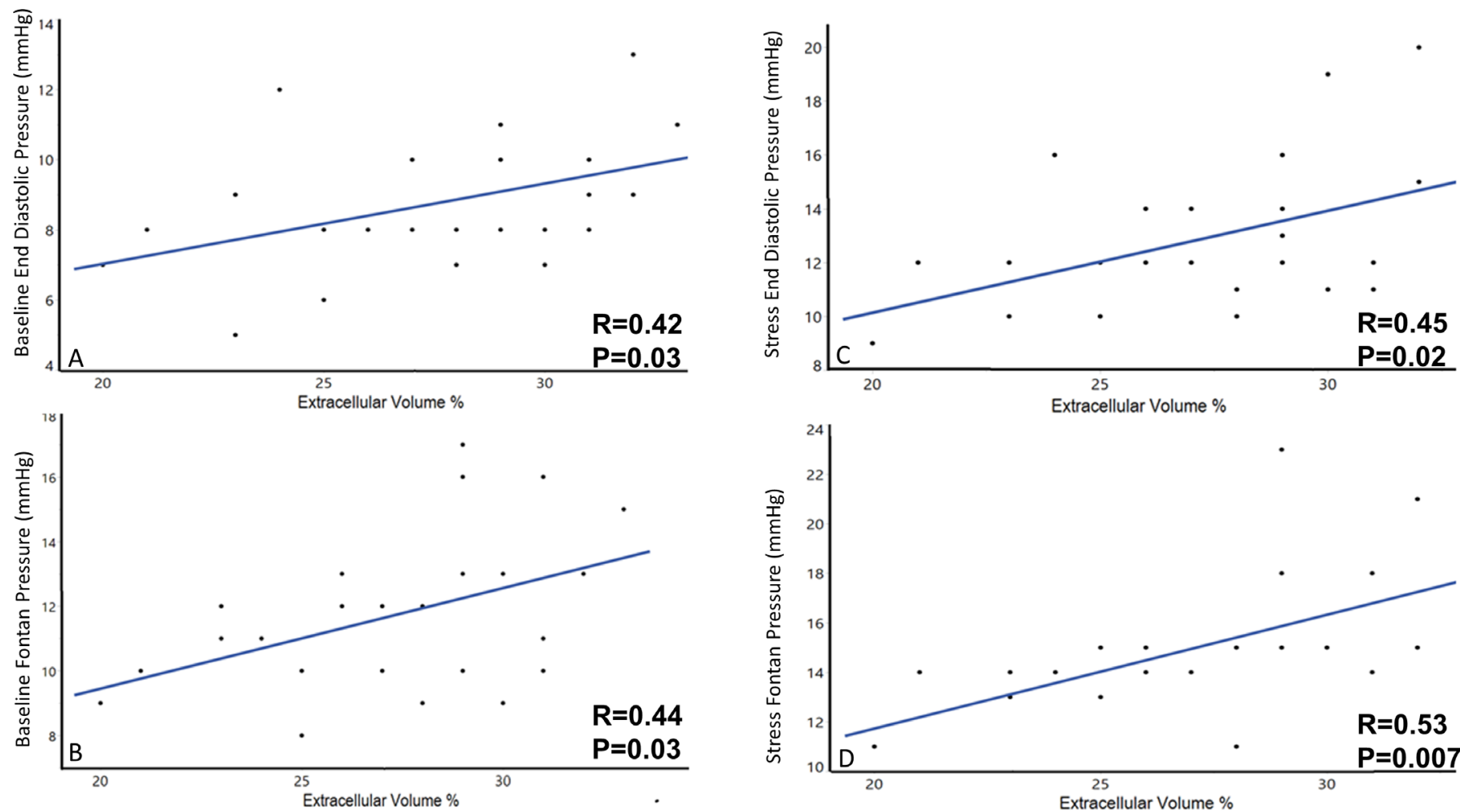

Figure 1 Scatter plots showing extracellular volume (ECV) and its associations with baseline and stress Fontan pressure. (A) Positive correlation between ECV and baseline end-diastolic pressure. (B) Positive correlation between ECV and baseline Fontan pressure. (C) Positive correlation between ECV and stress end-diastolic pressure. (D) Positive correlation between ECV and stress Fontan pressure.

\section{Liver stiffness and fibrosis measures}

Seventeen patients (68\% of the study population) had a liver MRE with stiffness measurements obtained; the mean liver stiffness was $4.1 \pm 0.7 \mathrm{kPa}$. There was a positive correlation between cardiac ECV and liver stiffness $(r=0.45, p=0.05)$. Patients categorised as DMF+ had significantly higher liver stiffness compared with DMF- patients $(\mathrm{p}=0.006)$ (table 2). Patients with elevated ( $>75$ th percentile) MMP1, MMP3, MMP8, MMP10, MMP13 and TIMP4 had higher liver stiffness measurements compared with patients with fibrosis biomarkers in the bottom three quartiles (figure 4, online supplemental table 3).

\section{DISCUSSION}

This study investigated the associations between myocardial fibrosis, invasive haemodynamics, liver stiffness and circulating biomarkers of fibrosis in adolescents and young adult patients with Fontan circulation. In this single-centre investigation, we found that ECV, a measure of myocardial fibrosis, was significantly associated with baseline and stress Fontan haemodynamics, including both Fontan pathway pressure and single ventricle EDP. Furthermore, ECV was associated with multiple serum biomarkers of fibrosis as well as MRE-derived liver stiffness. Patients with elevated circulating fibrosis biomarkers also had significantly increased liver stiffness. Our study is the first to relate myocardial fibrosis, diastolic dysfunction and increased liver stiffness in patients with a Fontan circulation and suggests that these maladaptive circulatory and end-organ changes may occur in the setting of a profibrotic milieu.

In an older Fontan population, Rathod $e t a l^{8}$ showed that LGE, a sign of macroscopic focal myocardial scarring, was common in Fontan patients and was associated with ventricular dysfunction and arrhythmia. No patient in our study was noted to have LGE, likely due to the younger age of this cohort, with a shorter duration of exposure to Fontan circulation. LGE imaging detects focal fibrosis and is based on differences in gadolinium volume of distribution between diseased and normal territories of the myocardium. ${ }^{14}$ Therefore, LGE is insensitive to the presence of diffuse myocardial fibrosis, which is likely to manifest at an earlier stage of pathologic myocardial remodelling in Fontan circulation. ${ }^{14}$ On the contrary, increased ECV correlates strongly with histologically quantified interstitial myocardial collagen. ${ }^{15}$

The measurement of ECV is becoming an important component in the assessment of many cardiovascular diseases and has been demonstrated to have significant prognostic implications in hypertrophic cardiomyopathy, pulmonary hypertension and HFpEF. ${ }^{16}$ Our group has previously suggested that Fontan failure may be a unique form of HFpEF, , what may be termed 'Fontan HFpEF', and the emerging data are supportive of this concept. In the first study of ECV in the Fontan circulation, Kato et a $\varphi^{P}$ showed that CMR-derived ECV determination is feasible and reproducible in Fontan patients and are associated with abnormal myocardial strain, but not ejection fraction 

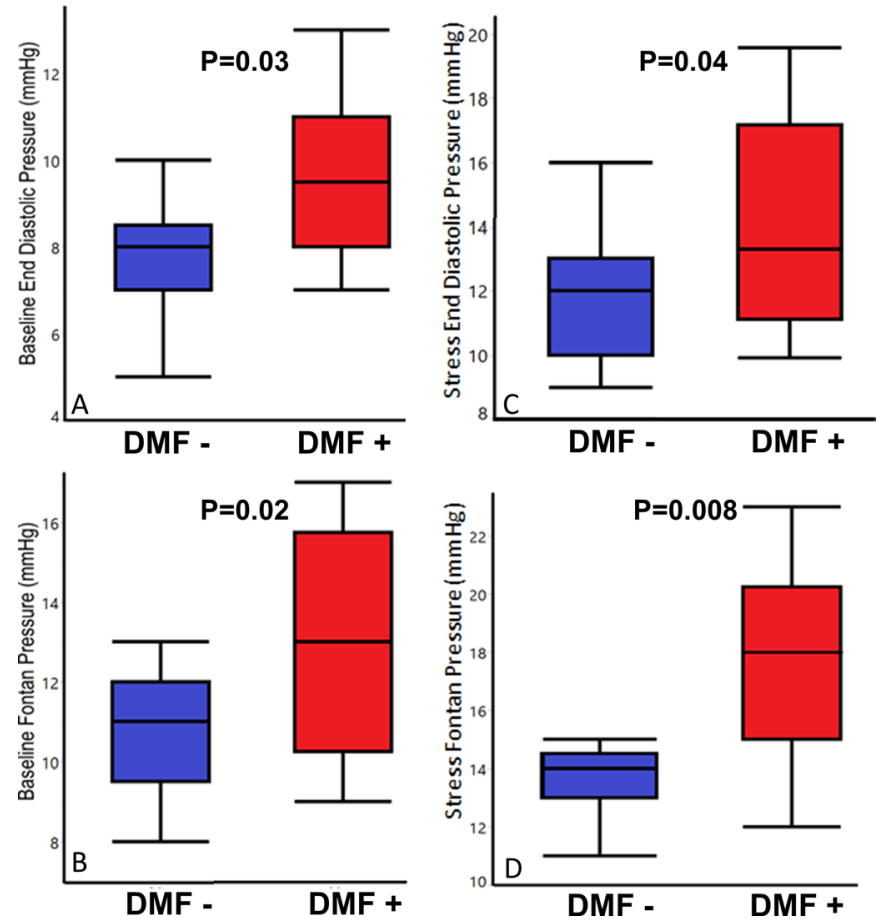

Figure 2 Tukey box plots showing resting and stress Fontan pressure in patients with and without diffuse myocardial fibrosis (DMF). (A) Baseline end-diastolic pressure (EDP) is higher in patients with DMF+ compared with patients with DMF-. (B) Stress Fontan pressure is higher in patients with DMF+ compared with patients with DMF-. (C) Stress EDP is higher in patients with DMF+ compared with patients with DMF-. (D) Stress Fontan pressure is higher in patients with DMF+ compared with patients with DMF-.

in children. In our older population, ECV correlated with systemic ventricular EDP both at baseline and after rapid volume expansion, suggesting that ECV is associated with diastolic dysfunction in the Fontan circulation. Diastolic dysfunction is an under-recognised aetiology of
Fontan circulatory failure. ${ }^{3} \mathrm{ECV}$ is therefore a promising non-invasive measure of adverse cardiac remodelling in Fontan patients.

There are some technical issues regarding the measurement of ECV that bears discussion. We were careful to report our ECV data in comparison with institutional norms, as it has been shown previously that such measurements can differ by MRI vendor and institution. Interestingly, however, myocardial ECV values in the present study were similar to those reported by Kato et al, ${ }^{9}$ despite utilising different CMR vendors. Furthermore, the reproducibility of myocardial ECV across institutions has been improving, as demonstrated in a recent large multicentre study of aortic stenosis. ${ }^{17}$ Therefore, it is likely that these data will be generalisable to the broader Fontan population.

While ECV was broadly associated with key Fontan haemodynamics in our population, the pathogenesis of diffuse myocardial fibrosis is likely multifactorial. Potential contributing factors include: altered preload at the different stages of single ventricle palliation, multiple exposures to cardiopulmonary bypass and circulatory arrest, ventricular volume overload due to systemic-topulmonary shunts, systemic-pulmonary collaterals and valvar regurgitation, and increased afterload, both focally (in cases of aortic arch obstruction) and broadly (due to chronically increased arterial stiffness and adverse vascular remodelling). ${ }^{18} 19$ As a result of these multiple factors, myocardial fibrosis is likely activated via multiple signalling and cellular pathways. ${ }^{20}$

Independent of the trigger, the generic response to activation of fibrosis pathways is the local and generalised release of several MMPs and their tissue inhibitors (TIMPs) involved in the degradation of collagen. ${ }^{12}$ MMPs and TIMPs are primarily produced by fibroblasts, which can be located in the myocardium, the

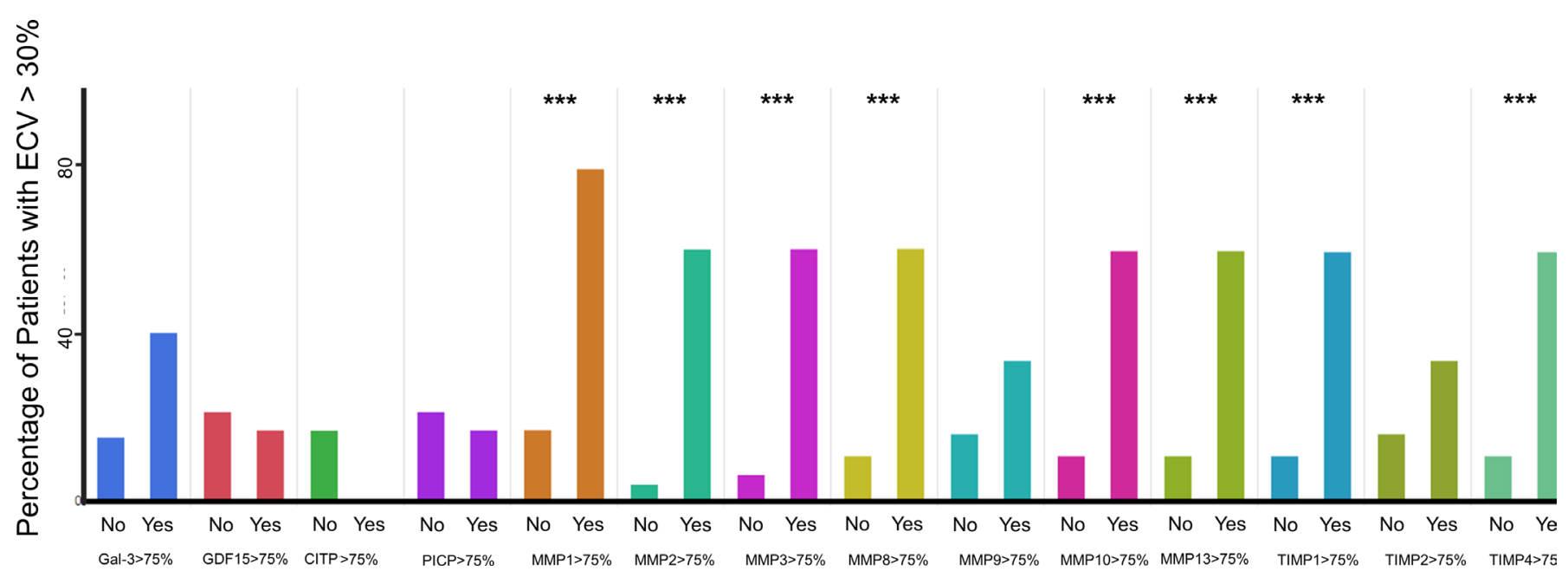

Figure 3 Circulating laboratory fibrotic biomarkers in patients with very high extracellular volume (ECV) $>30 \%$ compared with the rest of the patients. Each panel of two bars represents the levels of one biomarker (a different colour was used for each biomarker) in the group with and without ECV $>30 \%$. ${ }^{\star \star \star} \mathrm{p}<0.05$. GDF, growth differentiation factor; CITP, carboxy-terminal telopeptide of type I collagen; PICP, procollagen type I C-terminal propeptide; MMP, matrix metalloproteinase; TIMP, tissue inhibitor of metalloproteinase. 


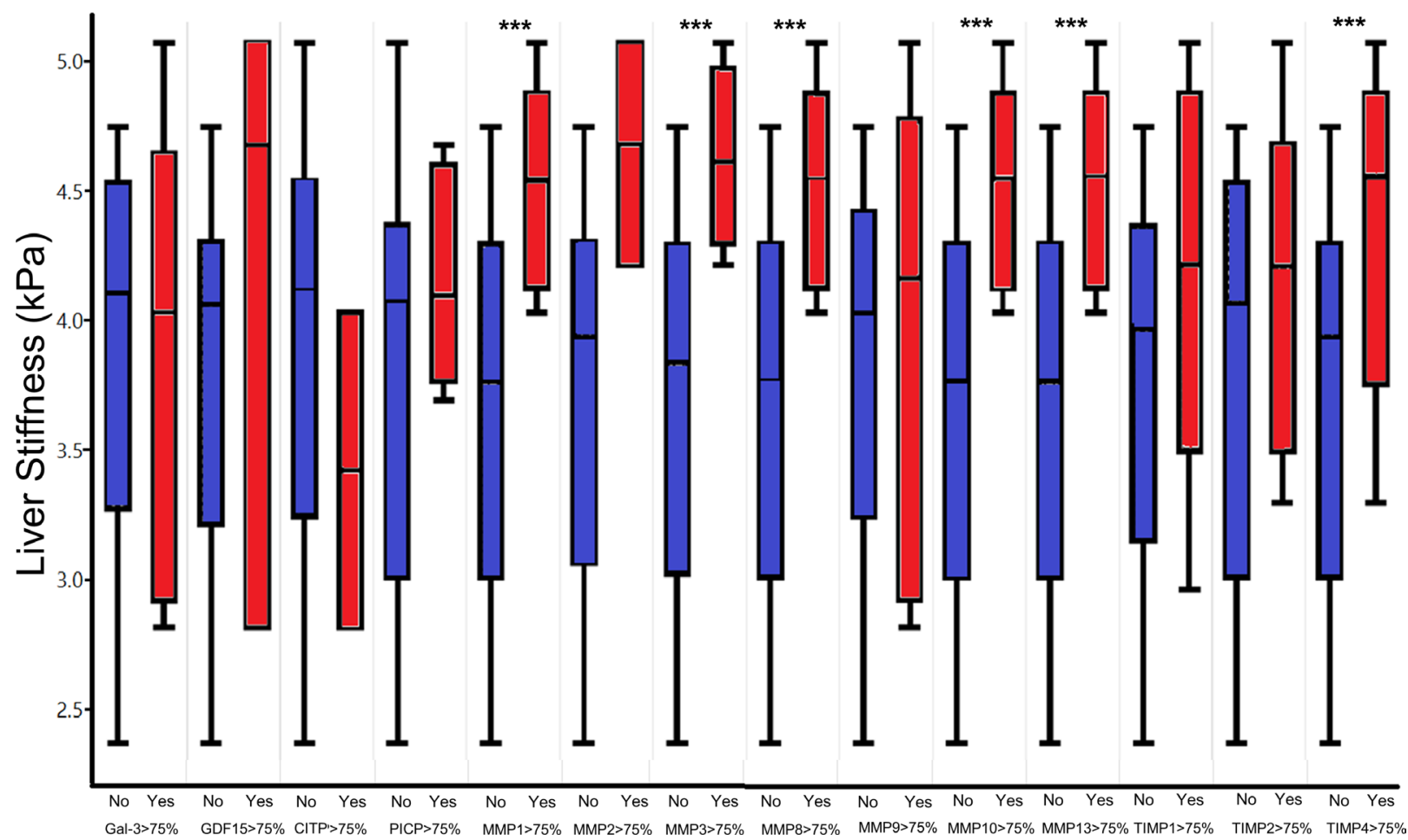

Figure 4 Liver stiffness measures in patients with elevated fibrotic biomarkers ( $>75$ th percentile) compared with the rest of the patients. ${ }^{* * *} \mathrm{p}$ value $<0.05$. GDF, growth differentiation factor; CITP, carboxy-terminal telopeptide of type I collagen; PICP, procollagen type I C-terminal propeptide; MMP, matrix metalloproteinase; TIMP, tissue inhibitor of metalloproteinase.

liver or other tissues. Their elevated levels may reflect the increased number of proliferating myocardial fibroblasts in other cardiomyopathies. ${ }^{21}$ While it remains to be determined whether these markers are cause or consequence (chicken or egg) of myocardial fibrosis, they are important markers of a profibrotic milieu in other conditions associated with myocardial fibrosis and, indeed, may improve in response to treatment with antifibrotic agents like mineralocorticoids antagonists. ${ }^{21}$ In our study, we related high levels of these profibrotic markers to the most severe phenotype of diffuse myocardial fibrosis in Fontan patients. Importantly, though, the elevation in serum fibrosis biomarkers correlated only with very high ECV - and not simply elevated ECV—suggesting that ECV may be more sensitive in the detection of myocardial fibrosis when compared with circulating biomarkers. Of note, many fibrosis biomarkers, including other collagen structural proteins (CITP, PICP), glycoproteins (galectin 3) and growth factors (GDF-15), were not elevated in the very high ECV group. Some of these biomarkers like GDF-15 have been shown recently to be associated with Fontan-related events in other studies. ${ }^{22}$ This may reflect heterogeneity of the fibrosis mechanisms present in the Fontan population in general, or this study cohort specifically.

We also found a positive association between ECV and liver shear stiffness measured by MRE. Increased liver stiffness is an imaging biomarker that correlates with increasing liver fibrosis but may also be present in the setting of liver congestion, prior to the development of fibrosis. ${ }^{1023}$ Our findings suggest that there is an association between hepatic and cardiac fibrosis in Fontan patients. There are several possible explanations for these findings: (1) progressive cardiac fibrosis and diastolic dysfunction result in adverse haemodynamics, which in turn hasten the progression in liver stiffness and fibrosis $^{25}$; (2) liver fibrosis results in activation of cardiac fibrosis as is well described in 'cirrhotic cardiomyopathy', which is also associated with a phenotype of diastolic dysfunction ${ }^{26}$; or (3) there is a profibrotic milieu in Fontan patients, which drives the development of cardiac and hepatic fibrosis and is associated with increased circulating MMPs and TIMPs. In support of the latter, MMPs and TIMPs have been shown in other populations to be elevated in both cardiac and hepatic fibrosis ${ }^{27}$ (figure 5). Elucidating the precise mechanism of both hepatic and myocardial fibrosis will require more detailed molecular studies and could provide therapeutic targets for diastolic dysfunction that could delay or prevent Fontan circulatory failure. $^{2} 28$

\section{Limitations}

While the 'deep phenotyping' of a Fontan cohort presented herein offers an in-depth analysis of fibrosis, there are important limitations present. First, the cohort size is small and derived from a single centre. Further multicentre studies will be needed to validate our results in other Fontan cohorts. Second, due to the use of CMR, patients with pacemakers and defibrillators were excluded. These devices are used in up to $13 \%$ of patients with Fontan circulation and are frequently associated with worse clinical outcomes; excluding these patients 


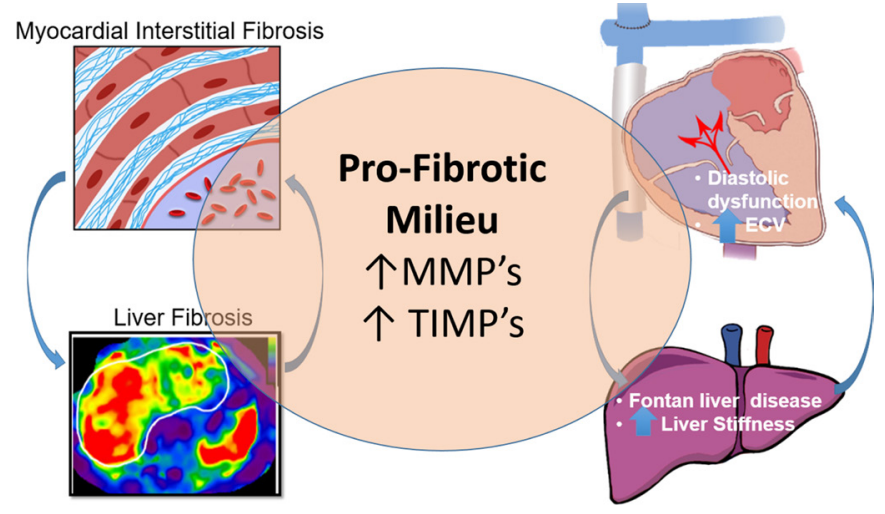

Figure 5 Graphical summary of the results and the potential relationship between hepatic and cardiac fibrosis. MMPs, matrix metalloproteinases; TIMPs, tissue inhibitor of metalloproteinases.

eliminates the opportunity to better assess if fibrosis is a mechanism of their deterioration. ${ }^{29}$ Third, sicker patients and patients with symptoms could be over-represented in this study, as these patients are more likely to be evaluated by cardiac catheterisation. ${ }^{30}$ However, during the study period, we implemented a Fontan management protocol that included the use of routine surveillance cardiac catheterisation, which we suspect helped to offset this concern. Furthermore, not all patients had liver MRE in this cohort although we found many significant associations in the small group who had MRE. Lastly, this crosssectional study did not evaluate for longitudinal changes in Fontan haemodynamics, myocardial fibrosis or liver stiffness. This will be the target of future research.

\section{CONCLUSION}

In adolescents and young adults with Fontan circulation, adverse resting and stress haemodynamics-indicative of diastolic dysfunction-are associated with myocardial fibrosis. The presence of elevated circulating MMPs and their tissue inhibitors are associated with both myocardial fibrosis and increased liver stiffness. These findings suggest the presence of a profibrotic milieu in some patients with Fontan circulation, which is associated with diffuse myocardial fibrosis, diastolic dysfunction and elevated liver stiffness.

\section{Author affiliations}

${ }^{1}$ Department of Pediatrics, The Heart Institute, Cincinnati Children's Hospital Medical Center, University of Cincinnati College of Medicine, Cincinnati, Ohio, USA ${ }^{2}$ Department of Cardiology, The Christ Hospital Health Network, Cincinnati, Ohio, USA

${ }^{3}$ Department of Pediatrics, Division of Cardiology, University of Alberta, Edmonton, Alberta, Canada

${ }^{4}$ Department of Radiology, Cincinnati Children's Hospital Medical Center, University of Cincinnati College of Medicine, Cincinnati, Ohio, USA

${ }^{5}$ Heart Institute, UPMC Children's Hospital of Pittsburgh, Department of Pediatrics, University of Pittsburgh Medical Center, Pittsburgh, Pennsylvania, USA

Acknowledgements We acknowledge Dr Elaine Urbina and Dr Philip Khoury for their assistance with this project.
Funding This work was supported by a grant from the Children's Heart Association of Cincinnati, PI: Bryan H Goldstein, MD. In addition, the Academic and Research Committee of Cincinnati Children's Hospital Medical Center provides grant support for the Fontan Multidisciplinary Clinic.

Competing interests None declared.

Patient consent for publication Not required.

Ethics approval This work was approved by Cincinnati Children's IRB \# 20159173.

Provenance and peer review Not commissioned; externally peer reviewed.

Data availability statement Data are available upon reasonable request.

Open access This is an open access article distributed in accordance with the Creative Commons Attribution Non Commercial (CC BY-NC 4.0) license, which permits others to distribute, remix, adapt, build upon this work non-commercially, and license their derivative works on different terms, provided the original work is properly cited, appropriate credit is given, any changes made indicated, and the use is non-commercial. See: http://creativecommons.org/licenses/by-nc/4.0/.

\section{ORCID iDs}

Tarek Alsaied http://orcid.org/0000-0002-3777-4822

Adam M Lubert http://orcid.org/0000-0001-6401-9980

Gruschen R Veldtman http://orcid.org/0000-0002-9336-2662

Bryan H Goldstein http://orcid.org/0000-0001-8508-9523

\section{REFERENCES}

1 Rychik J, Atz AM, Celermajer DS, et al. Evaluation and management of the child and adult with Fontan circulation: a scientific statement from the American heart association. Circulation 2019;140:CIR0000000000000696.

2 Alsaied T, Bokma JP, Engel ME, et al. Factors associated with longterm mortality after Fontan procedures: a systematic review. Heart 2017;103:104-10.

3 Budts W, Ravekes WJ, Danford DA, et al. Diastolic heart failure in patients with the Fontan circulation: a review. JAMA Cardiol 2020;5:590.

4 Averin K, Hirsch R, Seckeler MD, et al. Diagnosis of occult diastolic dysfunction late after the Fontan procedure using a rapid volume expansion technique. Heart 2016;102:1109-14.

5 Bing R, Dweck MR. Myocardial fibrosis: why image, how to image and clinical implications. Heart 2019;105:1832-40.

6 Kanagala P, Cheng ASH, Singh A, et al. Relationship Between Focal and Diffuse Fibrosis Assessed by CMR and Clinical Outcomes in Heart Failure With Preserved Ejection Fraction. JACC CardiovasC Imaging 2019;12:2291-301.

7 Niss O, Fleck R, Makue F, et al. Association between diffuse myocardial fibrosis and diastolic dysfunction in sickle cell anemia. Blood 2017:130:205-13.

8 Rathod RH, Prakash A, Powell AJ, et al. Myocardial fibrosis identified by cardiac magnetic resonance late gadolinium enhancement is associated with adverse ventricular mechanics and ventricular tachycardia late after Fontan operation. J Am Coll Cardiol 2010;55:1721-8.

9 Kato A, Riesenkampff E, Yim D, et al. Pediatric Fontan patients are at risk for myocardial fibrotic remodeling and dysfunction. Int $J$ Cardiol 2017;240:172-7.

10 Silva-Sepulveda JA, Fonseca Y, Vodkin I, et al. Evaluation of Fontan liver disease: correlation of transjugular liver biopsy with magnetic resonance and hemodynamics. Congenit Heart Dis 2019;14:600-8.

11 Seckeler MD, Hirsch R, Beekman RH, et al. A new predictive equation for oxygen consumption in children and adults with congenital and acquired heart disease. Heart 2015;101:517-24.

12 López B, González A, Ravassa S, et al. Circulating biomarkers of myocardial fibrosis: the need for a reappraisal. J Am Coll Cardiol 2015;65:2449-56.

13 Serai SD, Dillman JR, Trout AT. Spin-Echo Echo-planar imaging MR elastography versus gradient-echo MR elastography for assessment of liver stiffness in children and young adults suspected of having liver disease. Radiology 2017;282:761-70.

14 Moon JC, Messroghli DR, Kellman P, et al. Myocardial T1 mapping and extracellular volume quantification: a Society for cardiovascular magnetic resonance (SCMR) and CMR Working group of the European Society of cardiology consensus statement. J Cardiovasc Magn Reson 2013;15:92.

15 Flett AS, Hayward MP, Ashworth MT, et al. Equilibrium contrast cardiovascular magnetic resonance for the measurement of diffuse 
myocardial fibrosis: preliminary validation in humans. Circulation 2010;122:138-44.

16 Mordi IR, Singh S, Rudd A, et al. Comprehensive echocardiographic and cardiac magnetic resonance evaluation differentiates among heart failure with preserved ejection fraction patients, hypertensive patients, and healthy control subjects. JACC Cardiovasc Imaging 2018;11:577-85.

17 Everett RJ, Treibel TA, Fukui M, et al. Extracellular myocardial volume in patients with aortic stenosis. J Am Coll Cardiol 2020;75:304-16.

18 Rathod RH, Prakash A, Kim YY, et al. Cardiac magnetic resonance parameters predict transplantation-free survival in patients with Fontan circulation. Circ Cardiovasc Imaging 2014;7:502-9.

19 Goldstein BH, Urbina EM, Khoury PR, et al. Endothelial function and arterial stiffness relate to functional outcomes in adolescent and young adult Fontan survivors. J Am Heart Assoc 2016;5.

20 Heymans S, González A, Pizard A, et al. Searching for new mechanisms of myocardial fibrosis with diagnostic and/or therapeutic potential. Eur J Heart Fail 2015;17:764-71.

21 Polyakova V, Hein S, Kostin S, et al. Matrix metalloproteinases and their tissue inhibitors in pressure-overloaded human myocardium during heart failure progression. J Am Coll Cardiol 2004;44:1609-18.

22 Meyer SL, Wolff D, Ridderbos F-JS, et al. GDF-15 (growth differentiation factor 15) is associated with hospitalization and mortality in patients with a Fontan circulation. J Am Heart Assoc 2020;9:e015521.

23 Alsaied T, Possner M, Lubert AM, et al. Relation of magnetic resonance elastography to Fontan failure and portal hypertension. Am J Cardiol 2019;124:1454-9.

24 de Lange C, Reichert MJE, Pagano JJ, et al. Increased extracellular volume in the liver of pediatric Fontan patients. $J$ Cardiovasc Magn Reson 2019;21:39.

25 Wu FM, Opotowsky AR, Raza R, et al. Transient elastography may identify Fontan patients with unfavorable hemodynamics and advanced hepatic fibrosis. Congenit Heart Dis 2014;9:438-47.

26 Chayanupatkul M, Liangpunsakul S. Cirrhotic cardiomyopathy: review of pathophysiology and treatment. Hepatol Int 2014;8:308-15.

27 Roeb E. Matrix metalloproteinases and liver fibrosis (translational aspects). Matrix Biol 2018;68-69:463-73.

28 Book WM, Gerardin J, Saraf A, et al. Clinical phenotypes of Fontan failure: implications for management. Congenit Heart Dis 2016;11:296-308.

29 Anderson PAW, Sleeper LA, Mahony L, et al. Contemporary outcomes after the Fontan procedure: a pediatric heart network multicenter study. J Am Coll Cardiol 2008;52:85-98.

30 Fernandes SM, Alexander ME, Graham DA, et al. Exercise testing identifies patients at increased risk for morbidity and mortality following Fontan surgery. Congenit Heart Dis 2011;6:294-303. 